\title{
Wave Equation of Micro Element Centroid Velocity in Thermodynamic System
}

\author{
Yicheng Chen \\ College of Physical Science and Technology, Central China Normal University, Wuhan, China \\ Email: cyc4618@163.com
}

How to cite this paper: Chen, Y.C. (2021) Wave Equation of Micro Element Centroid Velocity in Thermodynamic System. Open Access Library Journal, 8: e8082. https://doi.org/10.4236/oalib.1108082

Received: October 14, 2021

Accepted: November 8, 2021

Published: November 11, 2021

Copyright $\odot 2021$ by author(s) and Open Access Library Inc.

This work is licensed under the Creative Commons Attribution International License (CC BY 4.0).

http://creativecommons.org/licenses/by/4.0/

\begin{abstract}
As we all know, it is Maxwell's electromagnetic wave equation and Hertz's electromagnetic wave experiment that make our mobile communication wonderful today! Since the electromagnetic wave has been widely used after research in the field of electromagnetism, is there a corresponding wave in the field of thermodynamics? Starting from the relevant equations in non-equilibrium thermodynamics works, in some special cases, the wave equation of micro element centroid velocity of thermodynamic system is obtained by pure vector analysis. It is expected that the solution of the nonlinear equation can more accurately describe the causes and images of weather in theory, and then guide the artificial regulation of weather! Hope to find this wave and widely serve mankind! Or discuss it and get a more perfect result.
\end{abstract}

\section{Subject Areas}

Thermodynamics

\section{Keywords}

Nonequilibrium Thermodynamics, Wave Equation

\section{1. 引言}

本文是一篇基础性的理论研究, 研究的灵感来自于麦克斯韦的电磁场波 动方程和 H. R. 赫兹的电磁波实验。当年麦克斯韦推导出了电磁场波动方程, 但不知道有什么用! 别人嘲笑他只是作了一场数学推导游戏! 若干年后, 赫 兹受麦克斯韦波动方程的启发, 产生出了电磁波, 但赫兹却感叹: “我认为 我所发现的无线电波，不会有任何实际应用。”[1] [2]正是这些不被人看好的 研究, 经过一代又一代科学工作者不厌其烦地深入探索, 才有了我们今天精 彩纷呈的移动通信时代, 无论是个人, 团体还是国家, 大家都有着深刻的体 
验!

既然电磁学领域经过研究之后得到了用途如此广泛的电磁波, 那么热力 学领域是否也存在一种相应的波! 正是在这种思想的指引下, 本着探索的心 态, 才在非平衡态热力学著作里找到有关方程, 从而完成本论文。

\section{2. 用矢量分析的方法得到非线性波动方程}

对于一个普通的热力学系统, 在各向同性流体中, 当粘滞性不明显地依 赖空间坐标时, 我们得到一个重要的特殊情况 [3]:

$$
\rho \frac{\mathrm{d} v}{\mathrm{~d} t}=-\operatorname{grad} p+\eta \Delta v+\left(\frac{1}{3} \eta+\eta_{v}\right) \operatorname{graddiv} v+\eta_{r} \operatorname{rot}(2 \omega-\operatorname{rot} v)
$$

式中 $\rho$ 是单位体积的质量(假设体系中只有单一组元), $v$ 是质心的速度, $p$ 是平衡压强, $\eta_{v}$ 为体积粘滞率, $\eta$ 是普通(或切变)粘滞率, $\eta_{r}$ 是转动粘滞率, $\omega$ 是流体中各点的组元粒子的平均角速度。这是除了包含切变与体积粘滞性 外, 还包含转动粘滞性的 Navier-Stokes 方程。

我们可将内角动量的平衡方程写成下列形式[4]

$$
\frac{\mathrm{d} \omega}{\mathrm{d} t}=\frac{-2 \eta_{r}}{\rho \Theta}(2 \omega-\operatorname{rot} \boldsymbol{v})
$$

式中量 $\Theta$ 是组元粒子的每单位质量的(平均)惯量矩即转动惯量。可以看出, (1) 与(2)两式组成了 $\boldsymbol{\omega}$ 与 $\boldsymbol{v}$ 耦合的微分方程组。

对于不可压缩流体，我们有 $\operatorname{div} v=0$ 。(1)式成为:

$$
\rho \frac{\mathrm{d} \boldsymbol{v}}{\mathrm{d} t}=-\operatorname{grad} p+\eta \Delta \boldsymbol{v}+\eta_{r} \operatorname{rot}(2 \omega-\operatorname{rot} \boldsymbol{v})
$$

对(2)式两边施行旋度运算[5]:

$$
\operatorname{rot} \frac{\mathrm{d} \omega}{\mathrm{d} t}=\operatorname{rot}\left(\frac{-2 \eta_{r}}{\rho \Theta}(2 \boldsymbol{\omega}-\operatorname{rot} \boldsymbol{v})\right)
$$

牛顿时空观告诉我们, 空间坐标与时间是相互独立的变量, 因此, 当一个函 数同时对空间坐标和时间进行求导时, 可将对空间坐标和时间的求导次序相 互交换, 于是(4)式的左边可写成: $\operatorname{rot} \frac{\mathrm{d} \omega}{\mathrm{d} t}=\frac{\mathrm{d}}{\mathrm{d} t} \operatorname{rot} \omega$, 而(4)式的右边稍作整理, 则(4)式成为:

$$
\frac{\mathrm{d}}{\mathrm{d} t} \operatorname{rot} \omega=\frac{2 \eta_{r}}{\rho \Theta}[\operatorname{rot}(\operatorname{rot} \boldsymbol{v})-2 \operatorname{rot} \omega]
$$

由于 $\operatorname{rot}(\operatorname{rot} v)=\operatorname{graddiv} v-\Delta v$, 以及 $\operatorname{div} v=0$, 所以(5)式成为:

$$
\frac{\mathrm{d}}{\mathrm{d} t} \operatorname{rot} \omega=\frac{2 \eta_{r}}{\rho \Theta}[-\Delta \boldsymbol{v}-2 \operatorname{rot} \omega]
$$

同样因为 $\operatorname{rot}(\operatorname{rot} v)=\operatorname{graddiv} v-\Delta v=-\Delta \boldsymbol{v}$, (1)式则成为:

$$
\rho \frac{\mathrm{d} \boldsymbol{v}}{\mathrm{d} t}=-\operatorname{grad} p+\eta \Delta \boldsymbol{v}+2 \eta_{r} \operatorname{rot} \omega+\eta_{r} \Delta \boldsymbol{v}
$$

从(7)式解得 $\operatorname{rot} \omega$ : 


$$
\operatorname{rot} \omega=\frac{\rho}{2 \eta_{r}} \frac{\mathrm{d} \boldsymbol{v}}{\mathrm{d} t}-\frac{\eta}{2 \eta_{r}} \Delta \boldsymbol{v}-\frac{1}{2} \Delta \boldsymbol{v}+\frac{1}{2 \eta_{r}} \operatorname{grad} p
$$

将(8)式代入(6)式, 整理得:

$$
\Delta v-\frac{\rho^{2} \Theta}{4 \eta \eta_{r}} \frac{\mathrm{d}^{2} \boldsymbol{v}}{\mathrm{d} t^{2}}=\frac{\rho}{\eta} \frac{\mathrm{d} \boldsymbol{v}}{\mathrm{d} t}+\frac{1}{\eta} \operatorname{grad} p-\frac{\rho \Theta\left(\eta+\eta_{r}\right)}{4 \eta \eta_{r}} \frac{\mathrm{d}}{\mathrm{d} t} \Delta \boldsymbol{v}+\frac{\rho \Theta}{4 \eta \eta_{r}} \frac{\mathrm{d}}{\mathrm{d} t} \operatorname{grad} p
$$

令 $n^{-2}=\frac{\rho^{2} \Theta}{4 \eta \eta_{r}}$, 则上方程为:

$$
\Delta \boldsymbol{v}-\frac{1}{n^{2}} \frac{\mathrm{d}^{2} \boldsymbol{v}}{\mathrm{d} t^{2}}=\frac{\rho}{\eta} \frac{\mathrm{d} \boldsymbol{v}}{\mathrm{d} t}+\frac{1}{\eta} \operatorname{grad} p-\frac{\rho \Theta\left(\eta+\eta_{r}\right)}{4 \eta \eta_{r}} \frac{\mathrm{d}}{\mathrm{d} t} \Delta \boldsymbol{v}+\frac{\rho \Theta}{4 \eta \eta_{r}} \frac{\mathrm{d}}{\mathrm{d} t} \operatorname{grad} p
$$

其中

$$
n=\frac{2}{\rho} \sqrt{\frac{\eta \eta_{r}}{\Theta}}
$$

是波速。(10)式就是热力学系统的非线性波动方程, 式中 $p$ 是已知的压强函 数, 求解该方程时, 要考虑重心实质时间微商: $\frac{\mathrm{d} \bar{v}}{\mathrm{~d} t}=\frac{\partial \bar{v}}{\partial t}+\bar{v} \cdot \mathrm{Grad} \overline{\boldsymbol{v}}$, 其中 “Grad” 表示对矢量作梯度运算; 而波速(11)式由流体的 $\rho 、 \eta 、 \eta_{r}$ 和转动 惯量 $\Theta$ 决定。

\section{3. 结论与展望}

本文从非平衡态热力学著作中的有关方程出发, 在一些特殊情况下通过 纯粹的矢量分析顺利地得到了热力学系统微元质心速度的波动方程, 但其解 答无疑是复杂的！期望该非线性方程式的解能从理论上主导性地描述天气的 成因及其图象并进而指引人工调控天气[6]！期望能找到这种波并广泛为人类 服务! 或者由此讨论开来而得到更完美的结果。

\section{致 谢}

感谢在本文写作的过程中, 姚端正, 李利华, 张明生, 俞云伟, 张子良, 宋姝月, 郭红等人士提供的帮助!

\section{Conflicts of Interest}

The author declares no conflicts of interest.

\section{References}

[1] [日]广重彻, 著. 电波的发现 [M]. 物理学史. 北京: 求实出版社, 1988.

[2] 中科院物理所. 一小伙发现了无线电波, 竟直呼没用? [EB/OL] https://mp.weixin.qq.com/s/uKNgjP28nfyQOXKUpfao5Q, 2021-01-27.

[3] 德格鲁脱, 梅休尔, 著. 第十二章: 粘滞流动与驰豫现象, (37)式[M]. 非平衡态热 力学. 陆全康, 译. 上海: 上海科学技术出版社, 1981.

[4] 德格鲁脱, 梅休尔, 著. 第十二章: 粘滞流动与弛豫现象, (32)式 $[M]$. 非平衡态热 力学. 陆全康, 译. 上海: 上海科学技术出版社.

[5] 郭硕鸿. 附录 I: 矢量分析 [M]. 电动力学. 第 2 版. 北京: 高等教育出版社, 1997. 
[6] 业百科/综合百科. 气象台如何测量气温 [EB/OL].

https://www.yebaike.com/22/2142334.html, 2020-11-06

\section{Appendix (Abstract \& Keywords in Chinese) \\ 热力学系统微元质心速度的波动方程}

摘要: 正如大家所知道的, 正是由于麦克斯韦的电磁场波动方程和赫兹的电 磁波实验, 才使得我们今天的移动通信精彩纷呈! 既然电磁学领域经过研究 之后得到了用途如此广泛的电磁波, 那么热力学领域是否也存在一种相应的 波? 本文从非平衡态热力学著作中的有关方程出发, 在一些特殊情况下通过 纯粹的矢量分析的方法得到了热力学系统微元质心速度的波动方程, 期望该 非线性方程式的解能从理论上更精准地描述天气的成因及其图象并进而指引 人工调控天气! 期望能找到这种波并广泛为人类服务! 或者由此讨论开来而 得到更完美的结果。

关键词: 非平衡态热力学, 波动方程 